\title{
NEW ALGEBRAS USING ADDITIVE ABELIAN GROUPS I
}

\author{
Seul Hee Choi
}

\section{Abstract}

The simple non-associative algebra $N\left(e^{A_{S}}, q, n, t\right)_{k}$ and its simple subalgebras are defined in the papers [1], [3], [4], [5], [6], [12]. We define the non-associative algebra $\overline{W N_{\left(g_{n}, \mathfrak{A}\right), m, s}}$ and its antisymmetrized algebra $\overline{W N_{\left(g_{n}, \mathfrak{A}\right), m, s_{B}}}-$. We also prove that the algebras are simple in this work. There are various papers on finding all the derivations of an associative algebra, a Lie algebra, and a non-associative algebra (see [3], [5], [6], [9], [12], [14], [15]). We also find all the derivations $\operatorname{Der}_{\text {anti }}\left(W N\left(e^{ \pm x^{r}}, 0,2\right)_{B}{ }^{-}\right)$of the antisymmetrized algebra $W N\left(e^{ \pm x^{r}}, 0,2\right)_{B}{ }^{-}$and every derivation of the algebra is outer in this paper.

\section{Preliminaries}

Let $\mathbb{F}$ be a field of characteristic zero (not necessarily algebraically closed). Throughout this paper, $\mathbb{N}$ and $\mathbb{Z}$ will denote the non-negative integers and the integers, respectively. Throughout the paper $\mathfrak{A}$ will denote an abelian group containing $\mathbb{Z}$. Let $\mathbb{F}\left[x_{1}, \cdots, x_{m+s}\right]$ be the polynomial ring with the variables $x_{1}, \cdots, x_{m+s}$. Let $g_{1}, \cdots, g_{n}$ be given polynomials in $\mathbb{F}\left[x_{1}, \cdots, x_{m+s}\right]$. For $n, m, s \in \mathbb{N}$, let us define the commutative, associative $\mathbb{F}$-algebra $\mathbb{F}_{\left(g_{n}, \mathfrak{L}\right), m, s}=\mathbb{F}\left[e^{ \pm g_{1}}, \cdots, e^{ \pm g_{n}}, x_{1}^{ \pm 1}, \cdots, x_{m}^{ \pm 1}\right.$, $\left.x_{m+1}, \cdots, x_{m+s}\right]$ which is called a stable algebra in the paper (see [10]

Received July 13, 2009. Accepted August 13, 2009.

Mathematics Subject Classification: Primary 17B25, 17B40.

Key Words: simple, stable algebra, antisymmetrized algebra, abelian, derivation. 
and [13]) with the standard basis

$$
\begin{gathered}
\left\{e^{a_{1} g_{1}} \cdots e^{a_{n} g_{n}} x_{1}^{i_{1}} \cdots x_{m+s}^{i_{m+s}} \mid a_{1}, \cdots, a_{n} \in \mathfrak{A}, i_{1}, \cdots, i_{m} \in \mathbb{Z}, i_{m+1}, \cdots,\right. \\
\left.1_{m+s} \in \mathbb{N}\right\}
\end{gathered}
$$

and with the obvious addition and the multiplication (see [10], [?], [14], [16], [17]) where $m+s \geq n . \partial_{w}, 1 \leq w \leq m+s$, denotes the usual partial derivative with respect to $x_{w}$ on $\mathbb{F}_{\left(g_{n}, \mathfrak{A}\right), m, s}$ formally. For partial derivatives $\partial_{u}, \cdots, \partial_{v}$ of $\mathbb{F}_{\left(g_{n}, \mathfrak{A}\right), m, s}$, the composition $\partial_{u}^{j_{u}} \circ \cdots \circ \partial_{v}^{j_{v}}$ of them is denoted by $\partial_{u}^{j_{u}} \cdots \partial_{v}^{j_{v}}$ where $j_{u}, \cdots, j_{v} \in \mathbb{N}$. Let $A$ be the set

$$
\left\{\partial_{u}^{j_{u}} \cdots \partial_{v}^{j_{v}} \mid j_{u}, \cdots, j_{v} \in \mathbb{N}, 1 \leq u, \cdots, v \leq m+s\right\} .
$$

Let us define the vector space $W N\left(\left(g_{n}, \mathfrak{A}\right), m, s\right)=W N\left(\left(g_{n}, \mathfrak{A}\right), m, s\right)_{A}$ over $\mathbb{F}$ which is spanned by the standard basis

$$
\left\{e^{a_{1} g_{1}} \cdots e^{a_{n} g_{n}} x_{1}^{i_{1}} \cdots x_{m+s}^{i_{m+s}} \partial_{u}^{j_{u}} \cdots \partial_{v}^{j_{v}} \mid a_{1}, \cdots, a_{n} \in \mathfrak{A}, i_{1}, \cdots, i_{m} \in \mathbb{Z},\right.
$$

(1) $\left.i_{m+1}, \cdots, i_{m+s} \in \mathbb{N}, j_{u}, \cdots, j_{v} \in \mathbb{N}, 1 \leq u, \cdots, v \leq m+s\right\}$

Thus we may define the multiplication $*$ on $W N\left(\left(g_{n}, \mathfrak{A}\right), m, s\right)$ as follows:

$$
\begin{aligned}
& e^{a_{11} g_{1}} \cdots e^{a_{1 n} g_{n}} x_{1}^{i_{11}} \cdots x_{m+s}^{i_{1, m+s}} \partial_{u}^{j_{u}} \cdots \partial_{v}^{j_{v}} * e^{a_{21} g_{1}} \cdots e^{a_{2 n} g_{n}} x_{1}^{i_{21}} \\
& \cdots x_{m+s}^{i_{2, m+s}} \partial_{h}^{j_{h}} \cdots \partial_{w}^{j_{w}}=e^{a_{11} g_{1}} \cdots e^{a_{1 n} g_{n}} x_{1}^{i_{11}} \cdots x_{m+s}^{i_{1, m+s}} \partial_{u}^{j_{u}} \\
& \cdots \partial_{v}^{j_{v}}\left(e^{a_{21} g_{1}} \cdots e^{a_{2 n} g_{n}} x_{1}^{i_{21}} \cdots x_{m+s}^{i_{2, m+s}}\right) \partial_{h}^{j_{h}} \cdots \partial_{w}^{j_{w}}
\end{aligned}
$$

for any basis elements $e^{a_{11} g_{1}} \cdots e^{a_{1 n} g_{n}} x_{1}^{i_{11}} \cdots x_{m+s}^{i_{1, m+s}} \partial_{u}^{j_{u}} \cdots \partial_{v}^{j_{v}}$ and $e^{a_{21} g_{1}} \ldots$ $e^{a_{2 n} g_{n}} x_{1}^{i_{21}} \cdots x_{m+s}^{i_{2, m+s}} \partial_{h}^{j_{h}} \cdots \partial_{w}^{j_{w}} \in W N\left(\left(g_{n}, \mathfrak{A}\right), m, s\right)$. Thus we can define the Weyl-type non-associative algebra $\overline{W N_{\left(g_{n}, \mathfrak{A}\right), m, s}}$ with the multiplication $*$ in $(2)$ and with the set $W N\left(\left(g_{n}, \mathfrak{A}\right), m, s\right)$ (see [12], [14], [18]). For $B \subset A$, let us define the the non-associative subalgebra $\overline{W N_{\left(g_{n}, \mathfrak{A}\right), m, s}} B$ of the non-associative algebra $\overline{W N_{\left(g_{n}, \mathfrak{A}\right), m, s}}$ spanned by

$$
\begin{aligned}
& \left\{e^{a_{1} g_{1}} \cdots e^{a_{n} g_{n}} x_{1}^{i_{1}} \cdots x_{s}^{i_{s}} \partial_{u}^{j_{u}} \cdots \partial_{v}^{j_{v}} \mid a_{1}, \cdots, a_{n}, i_{1}, \cdots, i_{m} \in \mathbb{Z},\right. \\
& \text { (3) } \left.\quad i_{m+1}, \cdots, i_{s}, j_{u}, \cdots, j_{v} \in \mathbb{N}, \partial_{u}^{j_{u}} \cdots \partial_{v}^{j_{v}} \in B, 1 \leq u, \cdots, v \leq m+s\right\}
\end{aligned}
$$

If we take $B=\left\{\partial_{u}^{0} \cdots \partial_{v}^{0}\right\}$, then the algebra $\overline{W N_{\left(g_{n}, \mathfrak{A}\right), m, s}}$ is the $\mathbb{F}$ algebra $F_{\left(g_{n}, \mathfrak{A}\right), m, s}$. This implies that the algebra $\overline{W N_{\left(g_{n}, \mathfrak{A}\right), m, s_{B}}}$ contains the polynomial ring, naturally. The simplicity of $\overline{W N_{\left(g_{n}, \mathfrak{A}\right), m, s}}$ is depending on the set $B$. It is well known that the non-associative algebra $W N_{\left(g_{n}, \mathfrak{A}\right), m, s}$ is simple, even though it has the right annihilator (see [7] and [8]). A non-associative algebra $\overline{W N_{\left(g_{n}, \mathfrak{A}\right), m, s_{B}}}$ is symmetric if there is a isomorphism induced by change of variables (see [3]). Throughout 
the paper we put $\partial^{1}=\partial$ and $\partial^{k}=\partial\left(\partial^{k-1}\right)$. The algebra $\overline{W N_{\left(g_{n}, \mathfrak{A}\right), m, s}}{ }_{B}$ is $\mathfrak{A}^{n}$-graded as follows:

$$
\overline{W N_{\left(g_{n}, \mathfrak{A}\right), m, s}}=\bigoplus_{\left(a_{1}, \cdots, a_{n}\right) \in \mathfrak{A}^{n}} N_{\left(a_{1}, \cdots, a_{n}\right)}
$$

where $N_{\left(a_{1}, \cdots, a_{n}\right)}$ is the vector subspace of $\overline{W N_{\left(g_{n}, \mathfrak{A}\right), m, s}}$ spanned by

$$
\begin{aligned}
& \left\{e^{a_{1} g_{1}} \cdots e^{a_{n} g_{n}} x_{1}^{i_{1}} \cdots x_{s}^{i_{s}} \partial_{u}^{j_{u}} \cdots \partial_{v}^{j_{v}} \mid i_{1}, \cdots, i_{m} \in \mathbb{Z}, i_{m+1}, \cdots, i_{s},\right. \\
& \left.j_{u}, \cdots, j_{v} \in \mathbb{N}, \partial_{u}^{j_{u}} \cdots \partial_{v}^{j_{v}} \in B, 1 \leq u, \cdots, v \leq m+s\right\} .
\end{aligned}
$$

\section{Simplicities and Isomorphisms}

Note 1. Since the algebra $\overline{W N_{\left(g_{n}, \mathfrak{A}\right), m, s}}$ is $\mathfrak{A}^{n}$-graded by (4), the $(0, \cdots, 0)$-homogeneous component $N_{(0, \cdots, 0)}$ of the algebra $\overline{W N_{\left(g_{n}, \mathfrak{A}\right), m, s}}$ is the simple subalgebra $\overline{W N_{0, m, s}}$.

Lemma 1. Every non-zero element of the subalgebra $\overline{W N_{0, m, s}}$ of the algebra $\overline{W N_{\left(g_{n}, \mathfrak{A}\right), m, s_{B}}}$ is full, i.e., an ideal which contains the element is the algebra $\overline{W N_{\left(g_{n}, \mathfrak{A}\right), m, s}}$.

Proof. Since the subalgebra $\overline{W N_{0, m, s}}$ of the algebra $\overline{W N_{\left(g_{n}, \mathfrak{A}\right), m, s}}$ is simple, every non-zero element of $\overline{W N_{0, m, s}}$ is full in the algebra $\overline{W N_{0, m, s_{B}}}$. Thus it is easy to prove that the element is full in the algebra $\overline{W N_{\left(g_{n}, \mathfrak{A}\right), m, s}}$. This completes the proof of the lemma.

Theorem 1. The algebra $\overline{W N_{\left(g_{n}, \mathfrak{A}\right), m, s}}$ is simple and the matrix ring $M_{m+s}(\mathbb{F})$ is a subalgebra of $\overline{W N_{\left(g_{n}, \mathfrak{A}\right), m, s_{B}}}$ (see [12]).

Proof. By Lemma 1, every non-zero element of the subalgebra $\overline{W N_{0, m, s}} B$ of $\overline{W N_{\left(g_{n}, \mathfrak{A}\right), m, s}}$ is full in the algebra $\overline{W N_{\left(g_{n}, \mathfrak{A}\right), m, s}}$. This implies that since the algebra is $\mathfrak{A}^{n}$-graded, it is easy to prove that every non-zero element of the algebra $\overline{W N_{\left(g_{n}, \mathfrak{A}\right), m, s}}$ is full. This implies that the algebra is simple. This completes the proof of the theorem.

Theorem 2. The antisymmetrized algebra ${\overline{W N_{\left(g_{n}, \mathfrak{A}\right), m, s}}}_{B}$ of the algebra $\overline{W N_{\left(g_{n}, \mathfrak{A}\right), m, s}}$ is simple and the Lie algebra $s l_{m+s}(\mathbb{F})$ is a subalgebra of $\overline{W N_{\left(g_{n}, \mathfrak{A}\right), m, s} B}-$ (see [12]) where $B=\left\{\partial_{u} \mid 1 \leq u \leq m+s\right\}$.

Proof. Since the algebra is $\mathfrak{A}^{n}$-graded and the derived algebra $\left[\overline{W N_{\left(g_{n}, \mathfrak{A}\right), m, s_{B}}}-\overline{W N_{\left(g_{n}, \mathfrak{A}\right), m, s}}{ }_{B}\right]$ is itself, by Lemma 1 , it is easy to 
prove that every non-zero element of the algebra $\overline{W N_{\left(g_{n}, \mathfrak{A}\right), m, s}}{ }_{B}$ is full. This implies that the algebra is simple. This completes the proof of the theorem.

Corollary 1. The Lie algebra ${\overline{W N_{\left(g_{n}, \mathfrak{A}\right), m, s}}}_{B}$ is simple and the Lie algebra $s l_{m+s}(\mathbb{F})$ is a subalgebra of $\overline{W N_{\left(g_{n}, \mathfrak{A}\right), m, s}}{ }^{-}$(see [12]) where $B=\left\{\partial_{u} \mid 1 \leq u \leq m+s\right\}$.

Proof. Since it is easy to prove that the algebra $\overline{W N_{\left(g_{n}, \mathfrak{A}\right), m, s_{B}}}{ }^{-}$is a Lie algebra. So the proof of the corollary is straightforward by Theorem 2. This completes the proof of the corollary.

Proposition 1. Let $B_{1}$ and $B_{2}$ be subsets of $\left\{\partial_{u}^{j_{u}} \cdots \partial_{v}^{j_{v}} \mid j_{u}, \cdots, j_{v} \in\right.$ $\mathbb{N}, 1 \leq u, \cdots, v \leq m+s\}$. If the sets $B_{1}$ and $B_{2}$ have different cardinalities, then the algebras $\overline{W N_{\left(g_{n}, \mathfrak{A}\right), m, s}} B_{1}$ and $\overline{W N_{\left(g_{n}, \mathfrak{A}\right), m, s}} B_{2}$ are not isomorphic.

Proof. If the algebras of the proposition are isomorphic, then they have isomorphic right annihilators. But they are not isomorphic by assumption. This implies that the algebras are not isomorphic. Therefore we have proven the proposition.

Theorem 3. If $\mathfrak{A}_{1}$ and $\mathfrak{A}_{2}$ are isomorphic groups, then the algebras $\overline{W N_{\left(g_{n}, \mathfrak{A}_{1}\right), m, s}}$ and $\overline{W N_{\left(g_{n}, \mathfrak{A}_{2}\right), m, s}}$ are isomorphic.

Proof. Let $\theta$ be an isomorphism from the group $\mathfrak{A}_{1}$ to $\mathfrak{A}_{2}$. For a standard basis element $e^{a_{1} g_{1}} \cdots e^{a_{n} g_{n}} x_{1}^{i_{1}} \cdots x_{m+s}^{i_{m+s}} \partial_{u}^{j_{u}} \cdots \partial_{v}^{j_{v}}$ of $\overline{W N_{\left(g_{n}, \mathfrak{A}_{1}\right), m, s}}$, if we define $\mathbb{F}$-linear map $\bar{\theta}$ from $\overline{W N_{\left(g_{n}, \mathfrak{A}_{1}\right), m, s}}$ to

$\overline{W N_{\left(g_{n}, \mathfrak{A}_{2}\right), m, s}}$ as follows:

$$
\begin{aligned}
& \bar{\theta}\left(e^{a_{1} g_{1}} \cdots e^{a_{n} g_{n}} x_{1}^{i_{1}} \cdots x_{m+s}^{i_{m+s}} \partial_{u}^{j_{u}} \cdots \partial_{v}^{j_{v}}\right)=e^{\theta\left(a_{1}\right) g_{1}} \cdots e^{\theta\left(a_{n}\right) g_{n}} x_{1}^{i_{1}} \cdots \\
& x_{m+s}^{i_{m+s}} \partial_{u}^{j_{u}} \cdots \partial_{v}^{j_{v}}
\end{aligned}
$$

then $\bar{\theta}$ can be linearly extended to an algebra homomorphism from $\overline{W N_{\left(g_{n}, \mathfrak{A}_{1}\right), m, s}}$ to $\overline{W N_{\left(g_{n}, \mathfrak{A}_{2}\right), m, s_{B}}}$. Since $\theta$ is onto and one to one, $\bar{\theta}$ is also bijective. This implies that $\bar{\theta}$ is an isomorphism. Therefore we have proven the theorem.

Theorem 4. If $\mathfrak{A}_{1}$ and $\mathfrak{A}_{2}$ are isomorphic groups, then the antisymmetrized algebras $\overline{W N_{\left(g_{n}, \mathfrak{A}_{1}\right), m, s}}{ }_{B}$ and $\overline{W N_{\left(g_{n}, \mathfrak{A}_{2}\right), m, s_{B}}}-$ are isomorphic. 
Proof. Since the following equalities $\left[\overline{W N_{\left(g_{n}, \mathfrak{A}_{1}\right), m, s}}-\overline{W N_{\left(g_{n}, \mathfrak{A}_{1}\right), m, s}}{ }_{B}\right]$ $=\overline{W N_{\left(g_{n}, \mathfrak{A}_{1}\right), m, s_{B}}}{ }^{-}$and $\left[\overline{W N_{\left(g_{n}, \mathfrak{A}_{2}\right), m, s}}{ }_{B}, \overline{W N_{\left(g_{n}, \mathfrak{A}_{2}\right), m, s}}{ }^{-}\right]$ $={\overline{W N_{\left(g_{n}, \mathfrak{A}_{2}\right), m, s}}}_{B}-$ hold, the proof of the theorem is similar to the proof of Theorem 3. This completes the proof of the theorem.

Corollary 2. If $\mathfrak{A}_{1}$ and $\mathfrak{A}_{2}$ are isomorphic groups and $B=\left\{\partial_{u} \mid 1 \leq\right.$ $u \leq m+s\}$, then the Lie algebras $\overline{W N_{\left(g_{n}, \mathfrak{A}_{1}\right), m, s}}{ }_{B}$ and $\overline{W N_{\left(g_{n}, \mathfrak{A}_{2}\right), m, s_{B}}}$ are isomorphic.

Proof. The proof of the corollary is straightforward by Theorem 4 . Let us omit its proof.

\section{Derivations of the antisymmetrized algebra $W N\left(e^{ \pm x^{r}}, 0,2\right)_{B}{ }^{-}$}

For this section, let us put $B=\left\{\partial_{1}, \partial_{2}, \partial_{1}^{2}, \partial_{2}^{2}\right\}, \mathfrak{A}=\mathbb{Z}$, and $g=x^{r}$. Note 2. For any basis elements $e^{p x^{r}} e^{q y^{s}} x^{i} y^{j} \partial_{1}, e^{p x^{r}} e^{q y^{s}} x^{i} y^{j} \partial_{1}^{2}, e^{p x^{r}} e^{q y^{s}}$ $x^{i} y^{j} \partial_{2}$, and $e^{p x^{r}} e^{q y^{s}} x^{i} y^{j} \partial_{2}^{2}$ of $W N\left(e^{ \pm x^{r}}, 0,2\right)_{B}{ }^{-}$, and for given $c_{1}, c_{2} \in \mathbb{F}$, if we define an $\mathbb{F}$-linear map $D_{c_{1}, c_{2}}$ from the algebra $W N\left(e^{ \pm x^{r}}, 0,2\right)_{B}$ to itself as follows:

$$
\begin{aligned}
& D_{c_{1}, c_{2}}\left(\partial_{u}^{v}\right)=0, \\
& D_{c_{1}, c_{2}}\left(e^{p x^{r}} e^{q y^{s}} x^{i} y^{j} \partial_{u}^{v}\right) \\
& =\delta_{1, u} \delta_{1, v} c_{1} p e^{p x^{r}} e^{q y^{s}} x^{i} y^{j} \partial_{1}+\delta_{1, u} \delta_{1, v} c_{2} q e^{p x^{r}} e^{q y^{s}} x^{i} y^{j} \partial_{1} \\
& +\delta_{1, u} \delta_{2, v} c_{1} p e^{p x^{r}} e^{q y^{s}} x^{i} y^{j} \partial_{1}^{2}+\delta_{1, u} \delta_{2, v} c_{2} q e^{p x^{r}} e^{q y^{s}} x^{i} y^{j} \partial_{1}^{2} \\
& +\delta_{2, u} \delta_{1, v} c_{1} p e^{p x^{r}} e^{q y^{s}} x^{i} y^{j} \partial_{2}+\delta_{2, u} \delta_{1, v} c_{2} q e^{p x^{r}} e^{q y^{s}} x^{i} y^{j} \partial_{2} \\
& +\delta_{2, u} \delta_{2, v} c_{1} p e^{p x^{r}} e^{q y^{s}} x^{i} y^{j} \partial_{2}^{2}+\delta_{2, u} \delta_{2, v} c_{2} q e^{p x^{r}} e^{q y^{s}} x^{i} y^{j} \partial_{2}^{2}
\end{aligned}
$$

then the map $D_{c_{1}, c_{2}}$ can be linearly extended to the antisymmetrized algebra derivation of

$W N\left(e^{ \pm x^{r}}, 0,2\right)_{B}{ }^{-}$where $1 \leq u, v \leq 2, \delta_{1, j}$ and $\delta_{2, j}$ are Kronecker delta and $1 \leq j \leq 2$ (see [6], [7], [9]).

Lemma 2. For any derivation $D \in \operatorname{Der}_{\text {anti }}\left(W N\left(e^{ \pm x^{r}}, 0,2\right)_{B}{ }^{-}\right)$, we have that

$$
D\left(\partial_{u}^{v}\right)=0, \quad D\left(x^{i} \partial_{u}^{v}\right)=0, \quad D\left(y^{j} \partial_{u}^{v}\right)=0, \quad D\left(x^{i} y^{j} \partial_{u}^{v}\right)=0
$$

hold where $1 \leq u, v \leq 2$. 
Proof. Let $D$ be the derivation in the lemma. Since the algebra $W N\left(e^{ \pm x^{r}}, 0,2\right)_{B}{ }^{-}$is $\mathbb{Z}$-graded, $D\left(\partial_{1}\right)$ and $D\left(\partial_{1}^{2}\right)$ are the sum of terms in different homogeneous components of $W N\left(e^{ \pm x^{r}}, 0,2\right)_{B}{ }^{-}$in (4). Thus $D\left(\partial_{1}\right)$ and $D\left(\partial_{1}^{2}\right)$ can be written as follows:

$$
\begin{aligned}
D\left(\partial_{1}\right)= & \sum_{i, j \geq 0} a_{i, j, 1} e^{p x^{r}} e^{q x^{s}} x^{i} y^{j} \partial_{1}+\sum_{i, j \geq 0} a_{i, j, 2} e^{p x^{r}} e^{q x^{s}} x^{i} y^{j} \partial_{2}+ \\
& \sum_{i, j \geq 0} a_{i, j, 3} e^{p x^{r}} e^{q x^{s}} x^{i} y^{j} \partial_{1}^{2}+\sum_{i, j \geq 0} a_{i, j, 4} e^{p x^{r}} e^{q x^{s}} x^{i} y^{j} \partial_{2}^{2}, \\
D\left(\partial_{1}^{2}\right)= & \sum_{i, j \geq 0} b_{i, j, 1} e^{p x^{r}} e^{q x^{s}} x^{i} y^{j} \partial_{1}+\sum_{i, j \geq 0} b_{i, j, 2} e^{p x^{r}} e^{q x^{s}} x^{i} y^{j} \partial_{2}+ \\
& \sum_{i, j \geq 0} b_{i, j, 3} e^{p x^{r}} e^{q x^{s}} x^{i} y^{j} \partial_{1}^{2}+\sum_{i, j \geq 0} b_{i, j, 4} e^{p x^{r}} e^{q x^{s}} x^{i} y^{j} \partial_{2}^{2}
\end{aligned}
$$

with appropriate coefficients. Since $\partial_{1}$ centralizes $\partial_{1}^{2}$, we have that

$$
\begin{aligned}
& {\left[\sum_{i, j \geq 0} a_{i, j, 1} e^{p x^{r}} e^{q x^{s}} x^{i} y^{j} \partial_{1}+\sum_{i, j \geq 0} a_{i, j, 2} e^{p x^{r}} e^{q x^{s}} x^{i} y^{j} \partial_{2}\right.} \\
& \left.+\sum_{i, j \geq 0} a_{i, j, 3} e^{p x^{r}} e^{q x^{s}} x^{i} y^{j} \partial_{1}^{2}+\sum_{i, j \geq 0} a_{i, j, 4} e^{p x^{r}} e^{q x^{s}} x^{i} y^{j} \partial_{2}^{2}, \partial_{1}^{2}\right] \\
& +\left[\partial_{1}, \sum_{i, j \geq 0} b_{i, j, 1} e^{p x^{r}} e^{q x^{s}} x^{i} y^{j} \partial_{1}+\sum_{i, j \geq 0} b_{i, j, 2} e^{p x^{r}} e^{q x^{s}} x^{i} y^{j} \partial_{2}\right. \\
& \left.+\sum_{i, j \geq 0} b_{i, j, 3} e^{p x^{r}} e^{q x^{s}} x^{i} y^{j} \partial_{1}^{2}+\sum_{i, j \geq 0} b_{i, j, 4} e^{p x^{r}} e^{q x^{s}} x^{i} y^{j} \partial_{2}^{2}\right] \\
& =-\sum_{i, j \geq 0} p^{2} r^{2} a_{i, j, 1} e^{p x^{r}} e^{q y^{s}} x^{i+2 r-2} y^{j} \partial_{1}-\sum_{i, j \geq 0} p r(i+r-1) a_{i, j, 1} e^{p x^{r}} \\
& e^{q y^{s}} x^{i+r-2} y^{j} \partial_{1}-\sum_{i \geq 1} p r i a_{i, j, 1} e^{p x^{r}} e^{q y^{s}} x^{i+r-2} y^{j} \partial_{1}-\sum_{i \geq 2} i(i-1) a_{i, j, 1} \\
& e^{p x^{r}} e^{q y^{s}} x^{i-2} y^{j} \partial_{1}-\sum_{i, j \geq 0} p^{2} r^{2} a_{i, j, 2} e^{p x^{r}} e^{q y^{s}} x^{i+2 r-2} y^{j} \partial_{2}-\sum_{i, j \geq 0} p r(i+r \\
& -1) a_{i, j, 2} e^{p x^{r}} e^{q y^{s}} x^{i+r-2} y^{j} \partial_{2}-\sum_{i \geq 1} p r i a_{i, j, 2} e^{p x^{r}} e^{q y^{s}} x^{i+r-2} y^{j} \partial_{2} \\
& -\sum_{i \geq 2} i(i-1) a_{i, j, 2} e^{p x^{r}} e^{q y^{s}} x^{i-2} y^{j} \partial_{2}-\sum_{i, j \geq 0} p^{2} r^{2} a_{i, j, 3} e^{p x^{r}} e^{q y^{s}} x^{i+2 r-2} y^{j} \partial_{1}^{2} \\
& -\sum_{i, j \geq 0} p r(i+r-1) a_{i, j, 3} e^{p x^{r}} e^{q y^{s}} x^{i+r-2} y^{j} \partial_{1}^{2}
\end{aligned}
$$




$$
\begin{aligned}
& -\sum_{i \geq 1} \operatorname{pria}_{i, j, 3} e^{p x^{r}} e^{q y^{s}} x^{i+r-2} y^{j} \partial_{1}^{2}-\sum_{i \geq 2} i(i-1) a_{i, j, 3} e^{p x^{r}} e^{q y^{s}} x^{i-2} y^{j} \partial_{1}^{2} \\
& -\sum_{i, j \geq 0} p^{2} r^{2} a_{i, j, 4} e^{p x^{r}} e^{q y^{s}} x^{i+2 r-2} y^{j} \partial_{2}^{2} \\
& -\sum_{i, j \geq 0} \operatorname{pr}(i+r-1) a_{i, j, 4} e^{p x^{r}} e^{q y^{s}} x^{i+r-2} y^{j} \partial_{2}^{2} \\
& -\sum_{i \geq 1} \operatorname{pri}_{i, j, 4} e^{p x^{r}} e^{q y^{s}} x^{i+r-2} y^{j} \partial_{2}^{2}-\sum_{i \geq 2} i(i-1) a_{i, j, 4} e^{p x^{r}} e^{q y^{s}} x^{i-2} y^{j} \partial_{2}^{2} \\
+ & \sum_{i, j \geq 0} \operatorname{prb}_{i, j, 1} e^{p x^{r}} e^{q y^{s}} x^{i+r-1} y^{j} \partial_{1}+\sum_{i \geq 1} i b_{i, j, 1} e^{p x^{r}} e^{q y^{s}} x^{i-1} y^{j} \partial_{1} \\
+ & \sum_{i, j \geq 0} \operatorname{prb}_{i, j, 2} e^{p x^{r}} e^{q y^{s}} x^{i+r-1} y^{j} \partial_{2}+\sum_{i \geq 1} i b_{i, j, 2} e^{p x^{r}} e^{q y^{s}} x^{i-1} y^{j} \partial_{2} \\
+ & \sum_{i, j \geq 0} \operatorname{pr}_{i, j, 3} e^{p x^{r}} e^{q y^{s}} x^{i+r-1} \partial_{1}^{2}+\sum_{i \geq 1} b_{i, j, 3} e^{p x^{r}} e^{q y^{s}} x^{i-1} \partial_{1}^{2} \\
& +\sum_{i, j \geq 0} \operatorname{pr}_{i, j, 4} e^{p x^{r}} e^{q y^{s}} x^{i+r-1} y^{j} \partial_{2}^{2}+\sum_{i \geq 1} i b_{i, j, 4} e^{p x^{r}} e^{q y^{s}} x^{i-1} y^{j} \partial_{2}^{2}=0
\end{aligned}
$$

with appropriate coefficients. Note that since the algebra is $\mathbb{Z}$-graded, it is enough to assume that non-zero terms of $D\left(\partial_{1}\right)$ and $D\left(\partial_{1}^{2}\right)$ are in the homogeneous components $N_{0}$ or $N_{p}$. By (6), we have that $a_{i, j, 1}, a_{i, j, 2}$, $a_{i, j, 3}, a_{i, j, 4}, b_{i, j, 1}, b_{i, j, 2} b_{i, j, 3}$, and $b_{i, j, 4}$ are zeroes, $i, j \geq 0$, and $D\left(\partial_{1}\right)$ and $D\left(\partial_{1}^{2}\right)$ are also zeroes. Similarly, we can prove that $D\left(\partial_{2}\right)$ and $D\left(\partial_{2}^{2}\right)$ are also zeroes. Since $\partial_{1}$ centralizes $x \partial_{1}$, we are able to prove that $D\left(x \partial_{1}\right)=$ $c_{0,0,1} \partial_{1}+c_{0,0,2} \partial_{2}+c_{0,0,3} \partial_{1}^{2}+c_{0,0,4} \partial_{2}^{2}$. Similarly, we also prove that $D\left(y \partial_{1}\right)=d_{0,0,1} \partial_{1}+d_{0,0,2} \partial_{2}+d_{0,0,3} \partial_{1}^{2}+d_{0,0,4} \partial_{2}^{2}$. Since $D\left(\left[\partial_{1}, x^{2} \partial_{1}\right]\right)=$ $\left[\partial_{1}, D\left(x^{2} \partial_{1}\right)\right]$, we are also able to prove that $D\left(x^{2} \partial_{1}\right)=2 c_{0,0,1} x \partial_{1}+$ $2 c_{0,0,2} x \partial_{2}+2 c_{0,0,3} x \partial_{1}^{2}+2 c_{0,0,4} x \partial_{2}^{2}+t_{1} \partial_{1}+t_{2} \partial_{2}+t_{3} \partial_{1}^{2}+t_{4} \partial_{2}^{2}$. Since $x \partial_{1}$ is an ad-diagonal element with respect to the element $x^{2} \partial_{1}$, we have that $c_{0,0,3}, t_{1}, t_{2}, t_{3}$, and $t_{4}$ are zeroes. This implies that $D\left(x \partial_{1}\right)=c_{0,0,1} \partial_{1}+$ $c_{0,0,2} \partial_{2}+c_{0,0,4} \partial_{2}^{2}$ and $D\left(x^{2} \partial_{1}\right)=2 c_{0,0,1} x \partial_{1}+2 c_{0,0,2} x \partial_{2}+2 c_{0,0,4} x \partial_{2}^{2}$ hold with appropriate coefficients. By $D\left(\left[y \partial_{1}, x \partial_{1}\right]\right)=D\left(y \partial_{1}\right)$, we have that $c_{0,0,2}=d_{0,0,2}=d_{0,0,3}=d_{0,0,4}=0$. This implies that

$$
\begin{aligned}
& D\left(x \partial_{1}\right)=c_{0,0,1} \partial_{1}+c_{0,04} \partial_{2}^{2}, D\left(y \partial_{1}\right)=d_{0,0,1} \partial_{1} \\
& D\left(x^{2} \partial_{1}\right)=2 c_{0,0,1} x \partial_{1}+2 c_{0,0,4} x \partial_{2}^{2}
\end{aligned}
$$

By $D\left(\left[\partial_{2}, y^{2} \partial_{1}\right]\right)=2 D\left(y \partial_{1}\right)$ and $D\left(\left[y^{2} \partial_{1}, x \partial_{1}\right]\right)=D\left(y^{2} \partial_{1}\right)$, we have that $c_{0,0,4}=0$ and $D\left(y^{2} \partial_{1}\right)=2 d_{0,0,1} y \partial_{1}+s_{1} \partial_{1}, s_{1} \in \mathbb{F}$. By $D\left(\left[y \partial_{1}, x^{2} \partial_{1}\right]\right)=$ $2 D\left(x y \partial_{1}\right)$, we have that $D\left(x y \partial_{1}\right)=d_{0,0,1} x \partial_{1}+c_{0,0,1} y \partial_{1}$. Since $D\left(\left[y \partial_{1}, x y\right.\right.$ 
$\left.\left.\partial_{1}\right]\right)=D\left(y^{2} \partial_{1}\right)$, we have that $D\left(y^{2} \partial_{1}\right)=2 d_{0,0,1} y \partial_{1}$. By (7), we have that

$$
\begin{aligned}
& D\left(x \partial_{1}\right)=c_{0,0,1} \partial_{1}, D\left(y \partial_{1}\right)=d_{0,0,1} \partial_{1}, D\left(x^{2} \partial_{1}\right)=2 c_{0,0,1} x \partial_{1} \\
& D\left(y^{2} \partial_{1}\right)=2 d_{0,0,1} y \partial_{1}, D\left(x y \partial_{1}\right)=d_{0,0,1} x \partial_{1}+c_{0,0,1} y \partial_{1}
\end{aligned}
$$

Since $D\left(\left[x^{i} \partial_{1}, x^{i+1} \partial_{1}\right]\right)=D\left(x^{2 i} \partial_{1}\right)$, by induction on $i$ of $x^{i} \partial_{1}$, we prove that $D\left(x^{2 i} \partial_{1}\right)=2 i c_{0,0,1} x^{2 i-1} \partial_{1}$. Similarly, we also prove that $D\left(x^{2 i-1} \partial_{1}\right)$ $=(2 i-1) c_{0,0,1} x^{2 i-2} \partial_{1}$. This implies that $D\left(x^{i} \partial_{1}\right)=i c_{0,0,1} x^{i-1} \partial_{1}$ for all $i$.

Since $D\left(\left[y^{i-1} \partial_{1}, x y \partial_{1}\right]\right)=D\left(y^{i} \partial_{1}\right)$, by induction on $i$ of $y^{i} \partial_{1}$, we also prove that $D\left(y^{i} \partial_{1}\right)=i d_{0,0,1} y^{i-1} \partial_{1}$. By $D\left(\left[\partial_{1}, x \partial_{1}^{2}\right]\right)=0$, we have that $D\left(x \partial_{1}^{2}\right)=g_{0,0,1} \partial_{1}+g_{0,0,2} \partial_{2}+g_{0,0,3} \partial_{1}^{2}+g_{0,0,4} \partial_{2}^{2}$. By $D\left(\left[\partial_{1}, x^{2} \partial_{1}^{2}\right]\right)=$ $2 D\left(x \partial_{1}^{2}\right)$ and $D\left(\left[x \partial_{1}, x^{2} \partial_{1}^{2}\right]\right)=2 D\left(x^{2} \partial_{1}^{2}\right)$, we can prove that $c_{0,0,1}=$ $g_{0,0,1}=g_{0,0,2}=g_{0,0,3}=g_{0,0,4}=0$. Thus, this means that $D\left(x^{i} \partial_{1}\right)=0$ and $D\left(x \partial_{1}^{2}\right)=D\left(x^{2} \partial_{1}^{2}\right)=0$. By $D\left(\left[x^{2} \partial_{1}, x^{i-1} \partial_{1}^{2}\right]\right)=(i-1) D\left(x^{i} \partial_{1}^{2}\right)-$ $2 D\left(x^{i-1} \partial_{1}\right)$ and by induction on $i$ of $x^{i} \partial_{1}^{2}$, we can prove that $D\left(x^{i} \partial_{1}^{2}\right)=0$ for all $i$. Similarly, we have that $D\left(y^{i} \partial_{2}\right)=D\left(y^{i} \partial_{2}^{2}\right)=0$. Since $\left[y \partial_{2}, y \partial\right]=D\left(y \partial_{1}\right)=d_{0,0,1} \partial_{1}$, we can prove that $d_{0,0,1}=0$. This implies that $D\left(y^{i} \partial_{1}\right)=0$. Similarly, we have that $D\left(x^{i} \partial_{2}\right)=0$. By $D\left(\left[\partial_{2}, y \partial_{1}^{2}\right]\right)=0$ and $D\left(\left[y \partial_{2}, y^{2} \partial_{1}^{2}\right]\right)=2 D\left(y^{2} \partial_{1}^{2}\right)$, we can prove that $D\left(y \partial_{1}^{2}\right)=D\left(y^{2} \partial_{1}^{2}\right)=0$. By induction on $i$ of $y^{i} \partial_{1}^{2}$, we have that $D\left(y^{i} \partial_{1}^{2}\right)=0$. Similarly, we have that $D\left(x^{i} \partial_{2}^{2}\right)=0$. Therefore we have proven the lemma.

Lemma 3. For any derivation $D \in \operatorname{Der}_{\text {anti }}\left(W N\left(e^{ \pm x^{r}}, 0,2\right)_{B}{ }^{-}\right)$, we have that

$$
D\left(e^{p x^{r}} x^{i} \partial_{u}^{v}\right)=c_{1} p e^{p x^{r}} x^{i} \partial_{u}^{v} \text { and } D\left(e^{q y^{s}} y^{j} \partial_{u}^{v}\right)=c_{2} q e^{q y^{s}} y^{j} \partial_{u}^{v}
$$

hold where $1 \leq u, v \leq 2$ and $c_{1}, c_{2} \in \mathbb{F}$.

Proof. Let $D$ be the derivation in the lemma. Assume that

$$
\begin{aligned}
D\left(e^{x^{r}} x^{k} \partial_{1}\right)= & \sum_{i, j \geq 0} u_{i, j, 1} e^{p x^{r}} e^{q y^{s}} x^{i} y^{j} \partial_{1}+\sum_{i, j \geq 0} u_{i, j, 2} e^{p x^{r}} e^{q y^{s}} x^{i} y^{j} \partial_{2} \\
& +\sum_{i, j \geq 0} u_{i, j, 3} e^{p x^{r}} e^{q y^{s}} x^{i} y^{j} \partial_{1}^{2}+\sum_{i, j \geq 0} u_{i, j, 4} e^{p x^{r}} e^{q y^{s}} x^{i} y^{j} \partial_{2}^{2},
\end{aligned}
$$


and

$$
\begin{aligned}
D\left(e^{x^{r}} x^{k+1} \partial_{1}\right)= & \sum_{i, j \geq 0} w_{i, j, 1} e^{p x^{r}} e^{q y^{s}} x^{i} y^{j} \partial_{1}+\sum_{i, j \geq 0} w_{i, j, 2} e^{p x^{r}} e^{q y^{s}} x^{i} y^{j} \partial_{2} \\
& +\sum_{i, j \geq 0} w_{i, j, 3} e^{p x^{r}} e^{q y^{s}} x^{i} y^{j} \partial_{1}^{2}+\sum_{i, j \geq 0} w_{i, j, 4} e^{p x^{r}} e^{q y^{s}} x^{i} y^{j} \partial_{2}^{2}
\end{aligned}
$$

hold with appropriate coefficients. By $D\left(\left[x \partial_{1}, e^{x^{r}} x^{k} \partial_{1}\right]\right)=r D\left(e^{x^{r}} x^{r+k} \partial_{1}\right)$ $+(k-1) D\left(e^{x^{r}} x^{k} \partial_{1}\right)$ and $D\left(\left[\partial_{1}, e^{x^{r}} x^{k+1} \partial_{1}\right]\right)=r D\left(e^{x^{r}} x^{r+k} \partial_{1}\right)+(k+$ 1) $D\left(e^{x^{r}} x^{k} \partial_{1}\right)$, we have that $D\left(\left[x \partial_{1}, e^{x^{r}} x^{k} \partial_{1}\right]\right)-(k-1) D\left(e^{x^{r}} x^{k} \partial_{1}\right)=$ $D\left(\left[\partial_{1}, e^{x^{r}} x^{k+1} \partial_{1}\right]\right)-(k+1) D\left(e^{x^{r}} x^{k} \partial_{1}\right)$. This implies that $w_{i+1, j, 1}=$ $u_{i, j, 1}, i, j \geq 0, w_{0, j, 1}=0, j \geq 0$, and $u_{i, j, l}=w_{i, j, l}=0, i, j \geq 0$, $2 \leq l \leq 4$. The followings

$$
\begin{aligned}
D\left(e^{x^{r}} x^{k+1} \partial_{1}\right) & =\sum_{i>1, j \geq 0} w_{i, j, 1} e^{p x^{r}} e^{q y^{s}} x^{i} y^{j} \partial_{1} \\
& =x\left(\sum_{i, j \geq 0} u_{i, j, 1} e^{p x^{r}} e^{q y^{s}} x^{i} y^{j} \partial_{1}\right)=x D\left(e^{x^{r}} x^{k} \partial_{1}\right)
\end{aligned}
$$

hold. We can write that $D\left(e^{x^{r}} x^{k} \partial_{1}\right)=x D\left(e^{x^{r}} x^{k-1} \partial_{1}\right)=x^{2} D\left(e^{x^{r}} x^{k-2} \partial_{1}\right)$ $=\cdots=x^{k} D\left(e^{x^{r}} \partial_{1}\right), u_{0, j, 1}=\cdots=u_{k-1, j, 1}=0, j \geq 0$, and $D\left(e^{x^{r}} \partial_{1}\right)=$ $\sum_{i>k, j>0} u_{i, j, 1} e^{p x^{r}} e^{q y^{s}} x^{i-k} y^{j} \partial_{1}$. By $D\left(\left[\partial_{1}, e^{x^{r}} \partial_{1}\right]\right)=r D\left(e^{x^{r}} x^{r-1} \partial_{1}\right)=$ $r x^{r-1} D\left(e^{x^{r}} \partial_{1}\right)$, we can prove that $p=1$ and $u_{i, j, 1}=0, i \geq k+1, j \geq 0$. So we have that $D\left(e^{x^{r}} \partial_{1}\right)=\sum_{j \geq 0} u_{k, j, 1} e^{x^{r}} e^{q y^{s}} y^{j} \partial_{1}$. By $D\left(\left[\partial_{2}, e^{x^{r}} \partial_{1}\right]\right)=$ 0 , we can prove that $D\left(e^{x^{r}} \partial_{1}\right)=u_{k, 0,1} e^{x^{r}} \partial_{1}=c_{1} e^{x^{r}} \partial_{1}$ with $c_{1}=u_{k, 0,1}$. By $D\left(\left[x \partial_{2}, e^{x^{r}} \partial_{1}\right]\right)=-D\left(e^{x^{r}} \partial_{2}\right)$, we have that $D\left(e^{x^{r}} \partial_{2}\right)=c_{1} e^{x^{r}} \partial_{2}$. Similarly, $D\left(e^{x^{r}} \partial_{2}^{2}\right)=c_{1} e^{x^{r}} \partial_{2}^{2}$. By $D\left(\left[x \partial_{1}^{2}, e^{x^{r}} \partial_{1}\right]\right)=r^{2} D\left(e^{x^{r}} x^{2 r-1} \partial_{1}\right)+r(r-$ 1) $D\left(e^{x^{r}} x^{2 r-1} \partial_{1}\right)-D\left(e^{x^{r}} \partial_{1}^{2}\right)$, we have that $D\left(e^{x^{r}} \partial_{1}^{2}\right)=c_{1} e^{x^{r}} \partial_{1}^{2}$. Similarly to the proof of $D\left(e^{x^{r}} \partial_{u}^{v}\right)=c_{1} e^{x^{r}} \partial_{u}^{v}, 1 \leq u, v \leq 2$, we can prove that $D\left(e^{y^{s}} \partial_{u}^{v}\right)=c_{2} e^{y^{s}} \partial_{u}^{v}$. By $D\left(\left[e^{x^{r}} \partial_{1}, e^{x^{x}} x \partial_{1}\right]\right)=D\left(e^{2 x^{r}} \partial_{1}\right)$, we have that $D\left(e^{2 x^{r}} \partial_{1}\right)=2 c_{1} e^{2 x^{r}} \partial_{1}$. By $D\left(\left[e^{x^{r}} \partial_{1}, e^{x^{r}} x^{i+1} \partial_{1}\right]\right)=(i+1) D\left(e^{2 x^{r}} x^{i} \partial_{1}\right)$, we also have that $D\left(e^{2 x^{r}} x^{i} \partial_{1}\right)=2 c_{1} e^{2 x^{r}} x^{i} \partial_{1}$. For proof of $p \in N$, we need to consider following two cases:

Case I. Put $p=2 m$. We have that $D\left(\left[e^{m x^{r}} \partial_{1}, e^{m x^{r}} x^{i+1} \partial_{1}\right]\right)=(i+$ 1) $D\left(e^{2 m x^{r}} x^{i} \partial_{1}\right)$. By induction on $p$ of $e^{p x^{r}} x^{i} \partial_{1}$, we are able to prove that

$$
D\left(e^{p x^{r}} x^{i} \partial_{1}\right)=p c_{1} e^{p x^{r}} x^{i} \partial_{1}
$$


Case II. Put $p=2 m+1$. The we have that

$$
\begin{aligned}
& D\left(\left[e^{m x^{r}} \partial_{1}, e^{(m+1) x^{r}} x^{i+1} \partial_{1}\right]\right) \\
& =r D\left(e^{(2 m+1) x^{r}} x^{r+i} \partial_{1}\right)+(i+1) D\left(e^{(2 m+1) x^{r}} x^{i} \partial_{1}\right)
\end{aligned}
$$

and

$$
\begin{aligned}
& D\left(\left[e^{m x^{r}} x^{i+1} \partial_{1}, e^{(m+1) x^{r}} \partial_{1}\right]\right) \\
& =r D\left(e^{(2 m+1) x^{r}} x^{r+i} \partial_{1}\right)-(i+1) D\left(e^{(2 m+1) x^{r}} x^{i} \partial_{1}\right) .
\end{aligned}
$$

By induction on $p$ of $e^{p x^{r}} x^{i} \partial_{1}$ and by subtracting (10) from (9), we are also able to prove that

$$
D\left(e^{p x^{r}} x^{i} \partial_{1}\right)=p c_{1} e^{p x^{r}} x^{i} \partial_{1} .
$$

By Case I and Case II, we have that

$$
D\left(e^{p x^{r}} x^{i} \partial_{1}\right)=p c_{1} e^{p x^{r}} x^{i} \partial_{1} .
$$

By $D\left(\left[e^{p x^{r}} \partial_{1}, x \partial_{1}^{2}\right]\right)=D\left(e^{p x^{r}} \partial_{1}^{2}\right)-p^{2} r^{2} D\left(e^{p x^{r}} x^{2 r-2} \partial_{1}\right)-p r(r-1) D\left(e^{p x^{r}}\right.$ $\left.x^{r-2} \partial_{1}\right)$, we have that $D\left(e^{p x^{r}} \partial_{1}^{2}\right)=p c_{1} e^{p x^{r}} \partial_{1}^{2}$. By $D\left(\left[x^{i+1} \partial_{1}^{2}, e^{p x^{r}} \partial_{1}\right]\right)=$ $p^{2} r^{2} D\left(e^{p x^{r}} x^{i+2 r-1} \partial_{1}\right)+p r(r-1) D\left(e^{p x^{r}} x^{i+r-1} \partial_{1}\right)-(i+1) D\left(e^{p x^{r}} x^{i} \partial_{1}^{2}\right)$, we also have that $D\left(e^{p x^{r}} x^{i} \partial_{1}^{2}\right)=p c_{1} e^{p x^{r}} x^{i} \partial_{1}^{2}$. By $D\left(\left[x \partial_{2}, e^{p x^{r}} x^{i} \partial_{1}\right]\right)=$ $-D\left(e^{p x^{r}} x^{i} \partial_{2}\right)$ and $D\left(\left[x \partial_{2}^{2}, e^{p x^{r}} x^{i} \partial_{1}\right]\right)=-D\left(e^{p x^{r}} x^{i} \partial_{2}^{2}\right)$, we have that

$$
D\left(e^{p x^{r}} x^{i} \partial_{2}^{v}\right)=p c_{1} e^{p x^{r}} x^{i} \partial_{2}^{v},
$$

where $1 \leq v \leq 2$. Similarly to the proof of the equality $D\left(e^{p x^{r}} x^{i} \partial_{u}^{v}\right)=$ $p c_{1} e^{p x^{r}} x^{i} \partial_{u}^{v}, 1 \leq u, v \leq 2$, we can prove that

$$
D\left(e^{q y^{s}} y^{j} \partial_{u}^{v}\right)=q c_{2} e^{p y^{s}} y^{j} \partial_{u}^{v}
$$

where $1 \leq u, v \leq 2$ and $c_{2} \in \mathbb{F}$. Therefore we have proven the lemma.

Lemma 4. For any derivation $D \in \operatorname{Der}_{\text {anti }}\left(W N\left(e^{ \pm x^{r}}, 0,2\right)_{B}{ }^{-}\right)$, we have that

$$
D\left(e^{p x^{r}} e^{q y^{s}} x^{i} y^{j} \partial_{u}^{v}\right)=c_{1} p e^{p x^{r}} e^{q y^{s}} x^{i} y^{j} \partial_{u}^{v}+c_{2} q e^{p x^{r}} e^{q y^{s}} x^{i} y^{j} \partial_{u}^{v}
$$

where $1 \leq u, v \leq 2$.

Proof. Let $D$ be the derivation in the lemma. By $D\left(\left[y^{j} \partial_{1}, e^{p x^{r}} x^{i+1} \partial_{1}\right]\right)$ $=\operatorname{pr} D\left(e^{p x^{r}} x^{i+r} y^{j} \partial_{1}\right)+(i+1) D\left(e^{p x^{r}} x^{i} y^{j} \partial_{1}\right)$ and $D\left(\left[x^{i+1} y^{j} \partial_{1}, e^{p x^{r}} \partial_{1}\right]\right)=$ $\operatorname{pr} D\left(e^{p x^{r}} x^{i+r} y^{j} \partial_{1}\right)-(i+1) D\left(e^{p x^{r}} x^{i} y^{j} \partial_{1}\right)$, we have that $D\left(e^{p x^{r}} x^{i} y^{j} \partial_{1}\right)=$ $c_{1} p e^{p x^{r}} x^{i} y^{j} \partial_{1}$. By $D\left(\left[e^{p x^{r}} x^{i} y^{j+1} \partial_{1}, e^{q y^{s}} \partial_{2}\right]\right)=-(j+1) D\left(e^{p x^{r}} e^{q y^{s}} x^{i} y^{j} \partial_{1}\right)$, we also have that

$$
D\left(e^{p x^{r}} e^{q y^{s}} x^{i} y^{j} \partial_{1}\right)=c_{1} p e^{p x^{r}} e^{q y^{s}} x^{i} y^{j} \partial_{1}+c_{2} q e^{p x^{r}} e^{q y^{s}} x^{i} y^{j} \partial_{1} .
$$


By $D\left(\left[y^{j} \partial_{1}, e^{p x^{r}} x^{i+1} \partial_{1}^{2}\right]\right)=\operatorname{pr} D\left(e^{p x^{r}} x^{i+r} y^{j} \partial_{1}^{2}\right)+(i+1) D\left(e^{p x^{r}} x^{i} y^{j} \partial_{1}^{2}\right)$ and $D\left(\left[x^{i+1} y^{j} \partial_{1}, e^{p x^{r}} \partial_{1}^{2}\right]\right)=\operatorname{pr} D\left(e^{p x^{r}} x^{i+r} y^{j} \partial_{1}^{2}\right)-(i+1) i D\left(e^{p x^{r}} x^{i-1} y^{j} \partial_{1}\right)$, we have that $D\left(e^{p x^{r}} x^{i} y^{j} \partial_{1}^{2}\right)=c_{1} p e^{p x^{r}} x^{i} y^{j} \partial_{1}^{2}$. Since $D\left(\left[e^{q y^{s}} \partial_{2}, e^{p x^{r}} x^{i} y^{j+1}\right.\right.$ $\left.\left.\partial_{1}^{2}\right]\right)=(j+1) D\left(e^{p x^{r}} e^{q y^{s}} x^{i} y^{j} \partial_{1}^{2}\right)$, we are able to prove that

$$
D\left(e^{p x^{r}} e^{q y^{s}} x^{i} y^{j} \partial_{1}^{2}\right)=c_{1} p e^{p x^{r}} e^{q y^{s}} x^{i} y^{j} \partial_{1}^{2}+c_{2} q e^{p x^{r}} e^{q y^{s}} x^{i} y^{j} \partial_{1}^{2} .
$$

Similarly, we can prove that

$$
\begin{aligned}
& D\left(e^{p x^{r}} e^{q y^{s}} x^{i} y^{j} \partial_{2}\right)=c_{1} p e^{p x^{r}} e^{q y^{s}} x^{i} y^{j} \partial_{2}+c_{2} q e^{p x^{r}} e^{q y^{s}} x^{i} y^{j} \partial_{2}, \\
& D\left(e^{p x^{r}} e^{q y^{s}} x^{i} y^{j} \partial_{2}^{2}\right)=c_{1} p e^{p x^{r}} e^{q y^{s}} x^{i} y^{j} \partial_{2}^{2}+c_{2} q e^{p x^{r}} e^{q y^{s}} x^{i} y^{j} \partial_{2}^{2}
\end{aligned}
$$

hold. Therefore we have proven the lemma.

Theorem 5. For any $D \in \operatorname{Der}_{\text {anti }}\left(W N\left(e^{ \pm x^{r}}, 0,2\right)_{B}{ }^{-}\right), D$ is the linear sum of the derivations $D_{c_{1}, c_{2}}$ as shown in Note 1 where $c_{1}, c_{2} \in \mathbb{F}$. The additive group $D \in \operatorname{Der}_{\text {anti }}\left(W N\left(e^{ \pm x^{r}}, 0,2\right)_{B}{ }^{-}\right)$is isomorphic to the additive group $\mathbb{F}^{2}$. Every derivation of the algebra $W N\left(e^{ \pm x^{r}}, 0,2\right)_{B}{ }^{-}$is outer.

Proof. The proofs of the theorem are straightforward by Lemma 4, Note 2 and the fact that the derivation of Note 2 cannot be inner. This completes the proof of the theorem.

Corollary 3. The dimension of $\operatorname{Der}_{\text {anti }}\left(W N\left(e^{ \pm x^{r}}, 0,2\right)_{B}{ }^{-}\right)$of the algebra $W N\left(e^{ \pm x^{r}}, 0,2\right)_{B}{ }^{-}$is two. For any derivation $D$ of $D e r_{a n t i}$ $\left(W N\left(e^{ \pm x^{r}}, 0,2\right)_{B}{ }^{-}\right), D\left(N_{0}^{\prime}\right)=0$ holds where $N_{0}^{\prime}$ is the zero-homogeneous component of $W N\left(e^{ \pm x^{r}}, 0,2\right)_{B}{ }^{-}$in (4) (see [2] and [8]).

Proof. The proofs of the corollary are straightforward by Theorem 3 and Note 2.

Remarks. In Proposition 1 , even if $B_{1}$ and $B_{2}$ have the same cardinalities, the algebras $\overline{W N_{\left(g_{n}, \mathfrak{A}\right), m, s}} B_{1}$ and $\overline{W N_{\left(g_{n}, \mathfrak{A}\right), m, s}} B_{2}$ cannot be isomorphic. It is interesting problem to find whether two algebras ${\overline{W N_{\left(g_{n}, \mathfrak{d}\right), m, s}}}_{B_{1}}$ and ${\overline{W N_{\left(g_{n}, \mathfrak{A}\right), m, s_{B_{2}}}}}$ are isomorphic or not. It is also interesting problem to find the automorphism group of an algebra $\overline{W N_{\left(g_{n}, \mathfrak{A}\right), m, s}} B_{1}$ and its all the derivations. 


\section{References}

[1] Mohammad H. Ahmadi, Ki-Bong Nam, and Jonathan Pakianathan, Lie admissible non-associative algebras, Algebra Colloquium, Vol. 12, No. 1, World Scientific, (March) 2005, 113-120.

[2] G. Brown, Properties of a 29-dimensional simple Lie algebra of characteristic three, Math. Ann., 261 (1982), no. 4, 487-492.

[3] Seul Hee Choi and Ki-Bong Nam, The Derivation of a Restricted Weyl Type NonAssociative Algebra, Vol. 28, No. 3, Hadronic Journal, 2005, 287-295.

[4] Seul Hee Choi, An algebra with right identities and its antisymmetrized algebra, Honam Mathematical Journal, Vol. 29, No. 2, 2007, 213-222..

[5] Seul Hee Choi and Ki-Bong Nam, "Weyl type non-associative algebra using additive groups I," Algebra Colloquium, Volume 14 (2007), 479-488, Number 3, 2007.

[6] Seul Hee Choi and Ki-Bong Nam, "Derivations of a restricted Weyl Type Algebra I", Rocky Mountain Math. Journals, Volume 37, Number 6, 2007, 67-84.

[7] Seul Hee Choi, Jongwoo Lee, and Ki-Bong Nam, "Derivations of a restricted Weyl type algebra containing the polynomial ring", Communication in Algebra, Volume 36, Issue 9 September 2008, 3435 - 3446.

[8] I. N. Herstein, Noncommutative Rings, Carus Mathematical Monographs, Mathematical Association of America, 100-101.

[9] T. Ikeda, N. Kawamoto and Ki-Bong Nam, A class of simple subalgebras of Generalized $W$ algebras, Proceedings of the International Conference in 1998 at Pusan (Eds. A. C. Kim), Walter de Gruyter Gmbh Co. KG, 2000, 189-202.

[10] V. G. Kac, Description of Filtered Lie Algebra with which Graded Lie algebras of Cartan type are Associated, Izv. Akad. Nauk SSSR, Ser. Mat. Tom, 38, 1974, 832-834.

[11] Naoki Kawamoto, Atsushi Mitsukawa, Ki-Bong Nam, and Moon-Ok Wang, The automorphisms of generalized Witt type Lie algebras, Journal of Lie Theory, 13 Vol(2), Heldermann Verlag, 2003, 571-576.

[12] Jongwoo Lee and Ki-bong Nam, "Non-Associative Algebras containing the Matrix Ring", Linear Algebra and its Applications Volume 429, Issue 1, 1 July 2008, Pages 72-78.

[13] Ki-Bong Nam, Generalized $W$ and H Type Lie Algebras, Algebra Colloquium 6:3, (1999), 329-340. 
[14] Ki-Bong Nam, On Some Non-Associative Algebras Using Additive Groups, Southeast Asian Bulletin of Mathematics, Vol. 27, Springer Verlag, 2003, 493-500.

[15] Ki-Bong Nam and Moon-Ok Wang, Notes on Some Non-Associative Algebras, Journal of Applied Algebra and Discrete Structured, Vol 1, No. 3, 159-164.

[16] D. P. Passman, Simple Lie algebras of Witt type, J. Algebra 206 (1998).

[17] A. N. Rudakov, Groups of Automorphisms of Infinite-Dimensional Simple Lie Algebras, Math. USSR-Izvestija, 3, 1969, 707-722.

[18] R. D. Schafer, Introduction to nonassociative algebras, Dover, 1995, 128-138.

Dept. of Mathematics,

Jeonju University,

Chon-ju 560-759, Korea, 